\title{
DIAMOND: A UK NATIONAL LIGHT SOURCE PROJECT
}

\author{
AA Chesworth, JA Clarke, GS Dobbing, DJ Holder, HL Owen, MW Poole, SL Smith, \\ VP Suller, A Wolski, CLRC Daresbury Laboratory, Warrington WA4 4AD, UK
}

\begin{abstract}
Modifications to the original racetrack lattice concept as used in the feasibility study of the DIAMOND light source project are presented here. Double bend achromat structures with both 16 and 20 cells are described, with 4 fold superperiodicity. The production of higher brightness beams through lower emittance is achieved by optics solutions having finite dispersion in the long straights. The implications of insertion device minimum gaps and of the beam energy spread are discussed with reference to the specification of the radiation spectrum required by users.
\end{abstract}

\section{PROJECT BACKGROUND}

In 1998 the UK government announced that funding had been allocated for a new X-ray synchrotron source, to replace the existing SRS at the Daresbury Laboratory. These funds will be jointly provided by the Government and the Wellcome Trust, which has a remit to fund research in the life sciences. The announced funding covers the 3 year period from April 1999, but it is assumed that later allocations will cover the full 6 year project timescale.

A review of the synchrotron radiation needs of the UK user community has recommended construction of a medium energy ring that complements the ESRF facilities in Grenoble; however for applications below about 50-100 $\mathrm{eV}$ an alternative national low energy source has been envisaged [1]. The $3 \mathrm{GeV}$ DIAMOND design has demonstrated the feasibility of an X-ray synchrotron source at a scale suitable for the needs of the UK user community. Designed originally as a 16 cell double bend achromat (DBA) racetrack [2], this has now been modified to a 4 fold superperiodicity to obtain improved flexibility and a better dynamic aperture [3].

Although a formal specification for DIAMOND is still in the process of being agreed, it is apparent that an important application of the facility will be in protein crystallography using samples of typical dimension $50 \mu \mathrm{m}$ and with very large cell dimensions. For this application high brightness photons at the selenium K-edge (12.7 $\mathrm{keV}$ ) are essential, with energies up to about $20 \mathrm{keV}$ also desirable, and several versions of the DIAMOND lattice have been studied to optimise performance against these criteria. High fluxes up to $50 \mathrm{keV}$ will be provided by multipole wigglers (MPW), with energies beyond this available from high field insertions; DIAMOND is also well optimised for high brightness output in the soft X-ray region down to $100 \mathrm{eV}$ or below. The extent to which even lower output energy $(5-50 \mathrm{eV})$ can meet a defined user case is still under investigation, but will not alter the overall DIAMOND optimisation.

Choosing between undulators or MPWs to deliver the required photon beams can have a significant influence on the facility specification, not least in the rf system demands. Additional factors are the ultimate minimum gap achievable with insertion devices and the electron beam energy. The study of these issues reported here indicates that a $3 \mathrm{GeV}, 20$ cell DBA lattice operated with finite dispersion in the long straights and with a circumference of about $400 \mathrm{~m}$ will produce the necessary high brightness beams up to $20 \mathrm{keV}$.

\section{RACETRACK LATTICE}

The original 16 cell racetrack lattice [2] consisted of 2 super-long cells together with 6 high and 8 low radial beta cells for injection, rf systems and more standard insertion devices. By independently powering the quadrupoles exact matching could be obtained between each type of cell. However the effect of the 2 super-long straights was to make the lattice fairly sensitive to errors and the 2-fold superperiodicity impacted strongly on the dynamic aperture achievable.

\section{FOUR-FOLD LATTICE}

With the continuing assessment of user requirements and after a review of the design of the racetrack machine it was decided that the basic DIAMOND design could be modified to obtain a number of improvements. Four-fold symmetry was selected with the prospect of better nonlinear lattice behaviour and the superstraights were shortened but able to accommodate all the accelerating cavities in one straight and injection components in another. Initial studies have been carried out on a DBA structure composed of 16 cells in four superperiods with straight section types in the following order: long short(low $\beta r)-\operatorname{short}($ high $\beta r)-\operatorname{short}($ low $\beta r$ ).

It is important in determining the final performance of this type of lattice that the flexibility to vary the straight section beta functions is assessed; this however is dependent on fully optimising the nonlinear properties of the lattice in each configuration. At the moment both the achromat and the short straights are based on those of the previous racetrack machine. The long straights have the same quadrupole arrangement but are now matched into 
an $11.8 \mathrm{~m}$ straight instead of the shorter straight. Preliminary nonlinear studies of this type of lattice at a variety of tune points have been carried out, and the results indicate a significant improvement in the ease of obtaining reasonable dynamic properties compared with the more demanding two-fold symmetric racetrack machine.

\section{CELL LATTICE}

As a national facility designed to satisfy the UK's needs well into the next century, the DIAMOND design must have the potential to deliver the number of sources and the quality of beam expected by users throughout its lifetime. Therefore studies have been carried out to investigate the performance and feasibility of a facility based upon a larger circumference, lower emittance ring. By extending the 16 cell four-fold symmetric lattice to 20 cells, it has been possible to confirm a reduction of emittance by about a factor of two, together with a useful increase in straight sections available for IDs from 14 to 18 .

The parameters for a potential 20 cell lattice are compared with those of the 16 cell lattice in Table 1. Although there is a significant increase in circumference over the original 16 cell lattices, this facility can fit comfortably on the Daresbury site. The table illustrates a four-fold symmetric solution but other options, such as a five-fold or even ten-fold symmetries, are still under consideration.

Table 1: Comparison of 16 and 20 cell lattices

\begin{tabular}{|l|c|c|}
\hline Lattice & 16 cell & 20 cell \\
\hline Energy [GeV] & 3.0 & 3.0 \\
\hline Circumference [m] & 329.2 & 396.8 \\
\hline Max length for IDs [m] & $\begin{array}{c}12 \times 4.5 \\
4 \times 8.0\end{array}$ & $\begin{array}{c}16 \times 4.5 \\
4 \times 8.0\end{array}$ \\
\hline Injection energy [GeV] & 3.0 & 3.0 \\
\hline Beam current [mA] & 300 & 300 \\
\hline Emittance; $\mathrm{h}, \mathrm{v}[\mathrm{nm} \mathrm{rad}]$ & $12.9,0.13$ & $7.8,0.08$ \\
\hline Source size $(\sigma)\left[\mu m^{2}\right]$ & & \\
Long straight; $\mathrm{h} \mathrm{x} \mathrm{v}$ & $497 \times 31.5$ & $249 \times 15.3$ \\
Short straight; $\mathrm{h} \mathrm{x} \mathrm{v}$ & $374 \times 10.2$ & $279 \times 11.2$ \\
\hline
\end{tabular}

The theoretical minimum emittance in a DBA can be reduced by a factor of 3 by allowing finite dispersion outside the achromatic arc. Studies with various realistic lattices have confirmed that it is possible in practice to obtain significant reduction in emittance under such conditions. In the particular case of a $3 \mathrm{GeV}, 20$ cell lattice with 4-fold symmetry, the emittance can be reduced from $8 \mathrm{~nm}$-rad to about 3-nm-rad, with consequent major benefits in the brightness. Table 2 shows the comparison between lattice parameters for zero and finite dispersion.
Table 2: Comparison of Zero/Finite Dispersion Settings

\begin{tabular}{|l|c|c|c|c|}
\cline { 2 - 5 } \multicolumn{1}{c|}{} & \multicolumn{2}{c|}{16 cell } & \multicolumn{2}{c|}{20 cell } \\
\cline { 2 - 5 } \multicolumn{1}{c|}{} & $\eta=0$ & $\eta \neq 0$ & $\eta=0$ & $\eta \neq 0$ \\
\hline Emittance $\mathcal{E}_{0}[\mathrm{~nm} \mathrm{rad}]$ & 12.9 & 4.95 & 7.8 & 2.67 \\
\hline $\mathrm{Mom}^{\mathrm{m}}$ compacn $\left[10^{-4}\right]$ & 8.73 & 8.42 & 4.64 & 4.45 \\
\hline Betatron tune $\mathrm{Q}_{x}$ & 16.41 & 16.40 & 20.46 & 20.48 \\
\hline Betatron tune $\mathrm{Q}_{y}$ & 9.85 & 9.88 & 11.93 & 11.29 \\
\hline Nat. chromat. $\xi_{x}$ & -63.60 & -46.63 & -69.9 & -69.4 \\
\hline Nat. chromat. $\xi_{y}$ & -22.25 & -22.63 & -21.6 & -27.6 \\
\hline Long straight $\eta_{x}[\mathrm{~m}]$ & 0 & 0.237 & 0 & 0.144 \\
\hline Short straight $\eta_{x}[\mathrm{~m}]$ & 0 & 0.185 & 0 & 0.155 \\
\hline Long straight $\beta_{x}[\mathrm{~m}]$ & 16.3 & 14.2 & 8.08 & 8.00 \\
\hline Long straight $\beta_{y}[\mathrm{~m}]$ & 6.55 & 9.00 & 3.05 & 2.10 \\
\hline Short straight $\beta_{x}[\mathrm{~m}]$ & 9.22 & 8.52 & 10.1 & 10.1 \\
\hline Short straight $\beta_{y}[\mathrm{~m}]$ & 0.690 & 0.753 & 1.63 & 1.20 \\
\hline
\end{tabular}

Representative lattice functions for the finite dispersion solution of the 20 cell version are shown in Fig. 1.

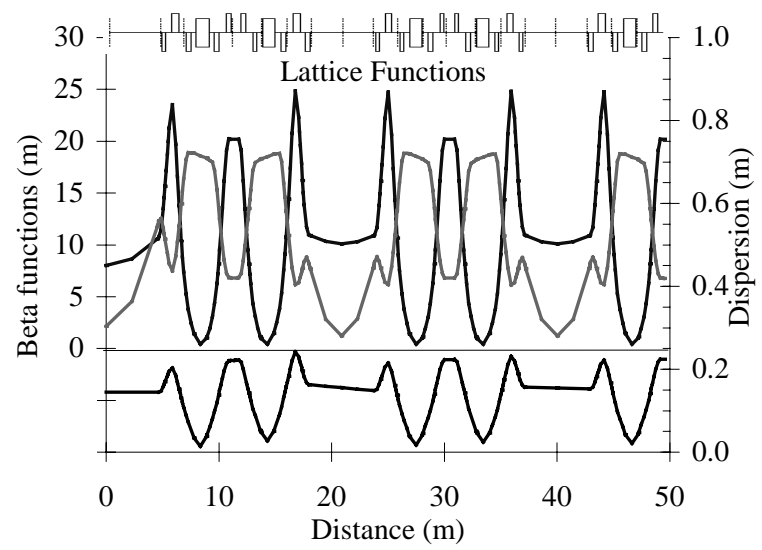

Figure 1: Finite dispersion lattice functions.

\section{PHOTON OUTPUT}

The photon output from DIAMOND will cover a large range, from infra-red to hard X-ray, although the source is optimised to provide undulator output from about $100 \mathrm{eV}$ to at least $5 \mathrm{keV}$ and multipole wiggler output to beyond $50 \mathrm{keV}$. The photon energy range between about 5 to 20 $\mathrm{keV}$ has recently taken on new importance with the greater emphasis on the user case for life sciences and, in particular, protein crystallography. This photon range is in the crossover region for use of multipole wigglers and undulators in a $3 \mathrm{GeV}$ source. The assumptions applied in earlier parameter feasibility studies, such as the minimum insertion device gap and the highest undulator harmonics to use, have been studied more carefully and revised. 


\subsection{Insertion Device Gap}

Early feasibility studies for DIAMOND assumed an initial insertion device gap of $20 \mathrm{~mm}$ with a gradual move towards smaller gaps (and therefore higher photon energy output from undulators) after the machine was commissioned and well understood. This approach is no longer possible as the source must now have undulator beamlines in the 5 to $20 \mathrm{keV}$ region operating from day one. Calculations have confirmed that beam lifetimes longer than 10 hours should be available with $5 \mathrm{~m}$ long vacuum vessels within an insertion device magnet gap of $15 \mathrm{~mm}$ or alternatively with shorter $2 \mathrm{~m}$ long vessels within a reduced magnet gap of $10 \mathrm{~mm}$. The results of representative ideal gas scattering calculations are shown in Fig. 2 and illustrate that at small gaps the elastic (coulomb) component dominates the losses; good lifetime is however still obtained for beam-stay-clear apertures well under $10 \mathrm{~mm}$, giving confidence in utilisation of small gap undulators. It is likely that in-vacuo solutions for DIAMOND insertion devices will also be explored.

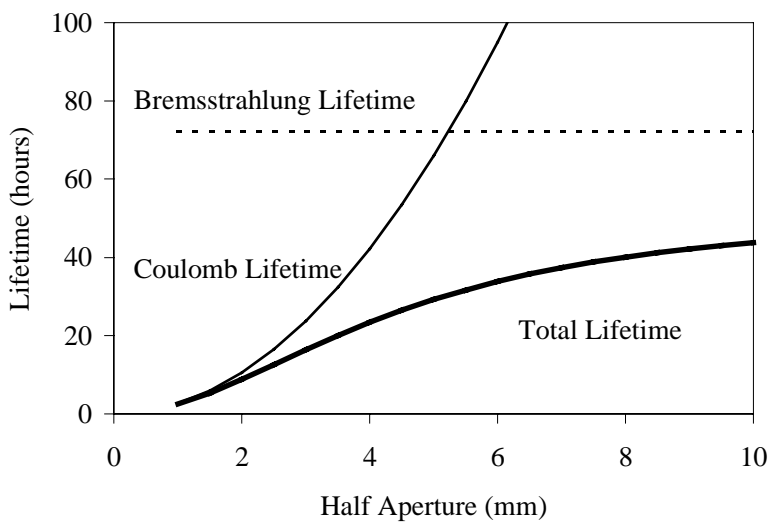

Figure 2: Gas scattering lifetime dependence on vertical aperture in a $5 \mathrm{~m}$ long insertion device vessel.

\subsection{Use of High Undulator Harmonics}

Undulators have generally been designed to utilise the first, third and occasionally fifth harmonics. For hard Xray beam lines requiring high brightness in DIAMOND it will be important to exploit harmonic output beyond the fifth and this has been carefully assessed. It is well known that at high harmonics factors such as magnet quality, beam emittance and energy spread have greater influence on the quality of the photon output. When these factors are taken into account the on-axis beam brightness can reduce by one or two orders of magnitude in some circumstances. However, calculations with SRW [4] have shown that if all of the photons in the central cone can be accepted by the beamline, then the flux reduction is generally less than a factor of two from the ideal, even allowing for an energy spread well above the natural value. A summary of such a result is given in Fig. 3 and an example $10 \mathrm{~mm}$ gap undulator tuning curve is shown in Fig. 4.

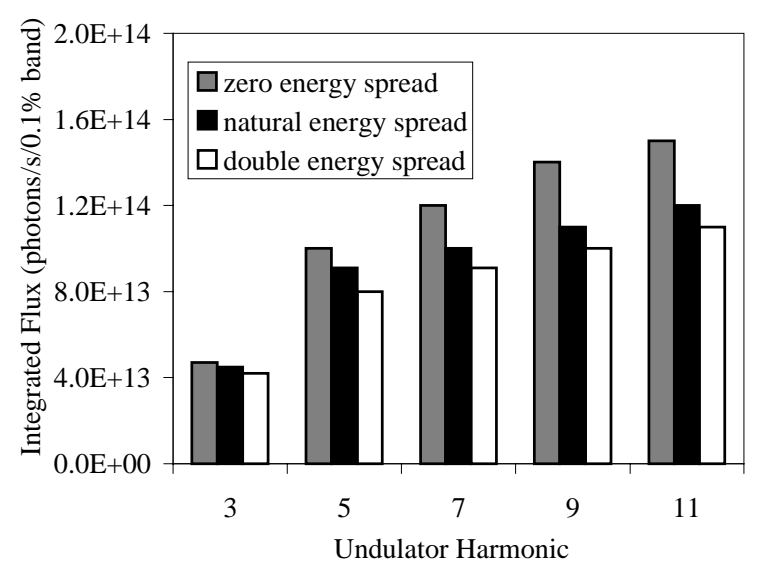

Figure 3: Undulator integrated flux at $10 \mathrm{~mm}$ gap.

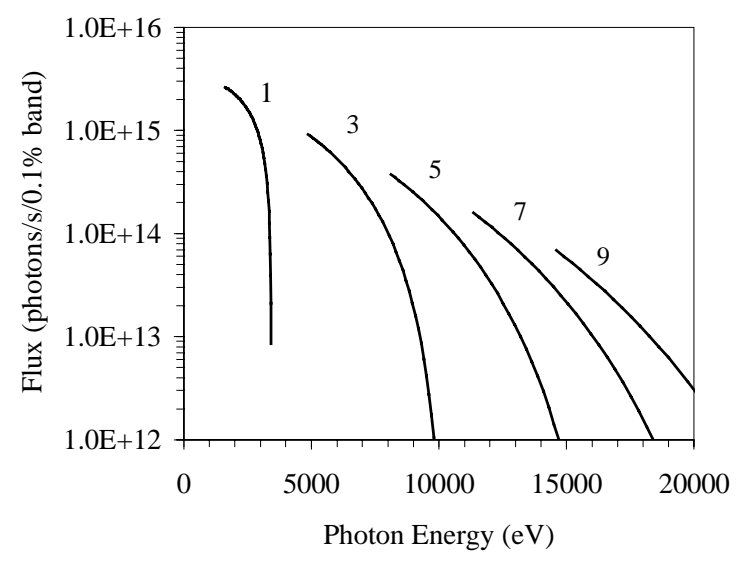

Figure 4: Example undulator tuning curve for a $25 \mathrm{~mm}$ period and $10 \mathrm{~mm}$ gap device.

DIAMOND can deliver high flux output from its undulators over a very wide energy range from below 100 $\mathrm{eV}$ up to $15 \mathrm{keV}$ and beyond. It is evident that at the higher energy end such undulators can be used in preference to the MPWs for topics such as protein crystallography, at least between 5 and $15 \mathrm{keV}$. This has major implications for the source specification, reducing the overall radiation power loading and also ensuring that an electron energy increase above $3 \mathrm{GeV}$ is unnecessary.

\section{REFERENCES}

[1] VP Suller; "A Source Design Strategy Providing 5eV-100keV Photons", Jour Synch Radn, 1, pp5-11, (1994).

[2] MW Poole \& VP Suller; "Design Progress on DIAMOND", Proc European Particle Accelerator Conference, Sitges, 1996.

[3] JA Clarke et al; "Update on the DIAMOND Light Source Project", Proc European Particle Accelerator Conference, Stockholm, 1998.

[4] O Chubar \& P Elleaume, "Accurate and Efficient Computation of Synchrotron Radiation in the Near Field Region", Proc European Particle Accelerator Conference, Stockholm, 1998. 